\title{
Transient Heat Hyperalgesia During Resolution of Ropivacaine Sciatic Nerve Block in the Rat
}

\author{
Lavinia M. Kolarczyk, MD* and \\ University of Pittsburgh, Department of Anesthesiology, A-1305 Scaife Hall, Pittsburgh, PA \\ 15213, Phone 412-383-5911, Fax 412-383-5466
}

Brian A. Williams, MD, MBA ${ }^{*}, \dagger$

Veterans Administration Pittsburgh Health System, University of Pittsburgh School of Medicine, Department of Anesthesiology

\begin{abstract}
Background-Preliminary studies using perineural sciatic ropivacaine in rat demonstrated unexpected heat hyperalgesia after block resolution. To better characterize the time course relative to mechanical anesthesia-analgesia, we tested the hypothesis that ropivacaine $0.5 \%$ leads to transient heat hyperalgesia in rat independent of mechanical nociception. We also evaluated functional toxicity (e.g., long-term hyperalgesia and/or tactile allodynia 2 weeks post-injection).

Methods-Under surgical exposure, left sciatic nerve block was performed in 2 groups of adult male rats - ropivacaine $(200 \mu \mathrm{L}, 5 \mathrm{mg} / \mathrm{mL}, \mathrm{n}=14)$ versus vehicle $(\mathrm{n}=11)$. The efficacy and duration of block was assessed with serial heat, mechanical (Randall-Selitto testing), and tactile (von Freylike monofilaments) tests; motor-proprioceptive (rotarod) and sedation tests were employed $1 \mathrm{hr}$ and $7 \mathrm{hr}$ post-injection. The presence of nerve injury was assessed by repeating the heat, tactile, and motor tests $12-14$ days post-injection.
\end{abstract}

Results-Ropivacaine-induced anesthesia was fully manifest at $1 \mathrm{hr}$ post-injection. At $3 \mathrm{hr}$ postinjection, heat hypersensitivity was present in the setting of resolved mechanical analgesia. All behavioral measures returned to baseline by 2 wk post-injection. There was no evidence of (i) behavioral sedation, (ii) persistent changes in heat or mechanical sensitivity, or (iii) persistent changes in proprioceptive-motor function at 12-14 days post-injection.

Conclusions-Ropivacaine $0.5 \%$ induces transient heat hyperalgesia in the setting of resolved mechanical analgesia, further suggestive of modality and/or nociceptive fiber specificity. Whether this finding partially translates to "rebound pain" after patients' nerve blocks wear off requires further study.

\section{Introduction}

With emerging evidence that volatile agents potentially contribute to transient postoperative hyperalgesia ${ }^{1,2,3}$, it is possible that thoughtful clinicians may choose to avoid general anesthesia with volatile agents in an effort to improve patient satisfaction. In light of this, it is imperative that peripheral nerve block (PNB) anesthesia also be investigated for possible postoperative hyperalgesia. Although counterintuitive, we are not aware of previous clinical

\footnotetext{
correspondence: kolarczyklm@upmc.edu.

*University of Pittsburgh School of Medicine, Department of Anesthesiology, Pittsburgh, Pennsylvania, United States.

$\dagger$ University of Pittsburgh School of Medicine, Center for Pain Research, Pittsburgh, Pennsylvania, United States.

This research was presented as a poster discussion at the 2010 American Society of Anesthesiologists Annual Meeting in San Diego,

California on October 17, 2010.
} 
or basic science reports of transient hyperalgesia attributed to PNB, but have long known that a "surge" of postoperative pain occurs very soon after a nerve block resolves (i.e., rebound pain) ${ }^{4}$ despite having ample circulating systemic opioids based on protocol-based dosing. Many questions remain unanswered about rebound pain: (i) is rebound pain partially attributable to local anesthetic nerve block resolution in the absence of a surgical stimulus?

(ii) Is rebound pain attributable simply to a barrage of previously-blocked nociceptive input? (iii) Could rebound pain be related to an interaction of these factors and/or some other inflammatory mediator? (iv) What role do specific peripheral nerve nociceptive fibers play in the development of rebound pain, and/or transient hyperalgesia? (v) Most importantly, can rebound pain and/or transient hyperalgesia be prevented? This manuscript will specifically address the fundamental question of whether there may be a component of rebound pain strictly attributable to local anesthetic nerve block resolution.

We introduce a refined rodent behavioral model that aims to inform potential roles of nociceptive fibers in hyperalgesia during and after resolution of a peripheral nerve block. We predict that this behavioral rat model may provide a basis for studying potential mechanisms of the clinical manifestation of "rebound pain." 4

In an earlier experiment in our laboratory ${ }^{1}$, ropivacaine-treated rats showed hyperalgesia to heat stimuli at 3-5 hr after perineural sciatic injection. The present study was therefore initiated in an attempt to: (i) verify hyperalgesia to heat stimulus after block resolution when studied as a specific aim; (ii) better quantify modality specificity of resolving perineural anesthesia versus a separate hyperalgesia phenomenon in response to mechanical and/or heat stimuli; (iii) determine whether persistent changes (perhaps reflecting potential focal neurotoxicity) occur.

\section{Materials and Methods}

Experiments were conducted using adult male Sprague-Dawley rats (mean weight $327 \mathrm{~g}$; Harlan, Indianapolis, IN) with University of Pittsburgh Institutional Animal Care and Use Committee (Pittsburgh, Pennsylvania, United States) approval. All animals were handled and cared for in accordance with the Guide for the Care and Use of Laboratory Animals (National Research Council). The rats were housed in the animal care facility with 12-hr alternating light-dark cycles (7:00 am dark-to-light transition), and unrestricted food and water intake for a minimum of $3 \mathrm{~d}$ before use in any experiment.

\section{Habituation, training, and behavioral testing protocol}

The protocol for habituation, training, and behavioral testing was carried out for $3 \mathrm{~d}$ preoperatively, which included handling and acclimation to testing rooms and testing apparatus.

Rats were first handled by the investigators for $5 \mathrm{~min}$, and were then transferred to a quiet behavioral testing room. The rats were then acclimated for minimum of $5 \mathrm{~min}$ to a raised, fenestrated stainless-steel table (which provided access to the plantar aspect of each hindpaw for application of von Frey monofilaments from below), followed by a minimum of $5 \mathrm{~min}$ acclimation on a glass thermal $\left(34^{\circ} \mathrm{C}\right)$ table apparatus (IITC $® 390$ Plantar Test Analgesia Meter, IITCR Life Science Instruments, inc., Woodland Hills, CA). Finally, rats were engaged in $10 \mathrm{~min}$ of rotating rod (rotarod) training (EZ-Rod 3.05, Accuscan Instruments, inc., Columbus, $\mathrm{OH})$. The rotarod software was programmed with standardized acceleration

\footnotetext{
${ }^{1}$ Williams BA, Gold MS, Gebhart GF, Tune AD, Yang CK: Motor and sensory behavioral models for rat sciatic nerve block ropivacaine versus saline vehicle (abstract), Proceedings of the 2009 Annual Meeting of the American Society Anesthesiologists. New Orleans, LA, American Society Anesthesiologists, 2009, pp A-576
} 
(to maximum $20 \mathrm{rpm}$ during the initial $60 \mathrm{sec}$ for training purposes, with a $20 \mathrm{rpm}$ plateau for $20 \mathrm{sec}$ ) and deceleration patterns (from $20 \mathrm{rpm}$ to zero during the last $40 \mathrm{sec}$ ).

On the day before scheduled surgery, baseline behavioral assessments of general activity, mechanical sensitivity, tactile sensitivity, thermal sensitivity, and motor-proprioception function (rotarod testing, as described above), were recorded. Activity monitoring (Med Associates Activity Monitor, v. 5, St. Albans, VT) entailed the use of infrared sensors to track rat movements while enclosed in a large, Plexiglas chamber. Mechanical sensitivity was evaluated using the Randall-Selitto paw withdrawal test ${ }^{5}$ (Analgesy-Meter - 37215, Ugo Basile biological research apparatus, Comerio, Italy), which employs a graded application of a calibrated mechanical force to the dorsal and plantar hindpaw; the mechanical force (grams) at which the rat withdrew the hindpaw was recorded. Tactile sensitivity was evaluated using monofilaments (19.6 to $588 \mathrm{mN}$ bending forces) using the Dixon up-down method ${ }^{6}$ (Touch Test Sensory Evaluator Kit, Stoelting, inc., Wood Dale, IL). For thermal testing, rats were acclimated for $5 \mathrm{~min}$ on the described thermal glass table apparatus. A calibrated heat lamp with a $50 \mathrm{~W}$ bulb beneath the glass table surface was used to provide a focal radiant thermal stimulus. The active intensity of the radiant heat source was set at $25 \%$ of maximal, and the maximum time of stimulus was limited to $20 \mathrm{sec}$. The temperature of the glass surface at the $20 \mathrm{sec}$ cut-off point was estimated to be 49.0 (SEM: $0.7)^{\circ} \mathrm{C}$, based on calibration of the apparatus before these experiments were conducted. Three heat stimuli were applied to each hind paw per testing session, with an inter-stimulus interval of $5 \mathrm{~min}$. Rats with 2 consecutive thermal tests of $20 \mathrm{sec}$ (i.e., cut-off) were not subjected to the third heat stimulus to avoid potential tissue damage to the hindpaw. If an animal ambulated during the heat stimulus, the test value was excluded.

\section{Randomization and blinding}

Rats were randomized using a random number generator to ropivacaine (RPV) versus vehicle (VEH) treatment groups. A laboratory technician was designated solely for creation of unlabeled syringes containing the injectate and for placing the syringes in a sealed, numbered envelope for administration by an investigator on the day of surgery. Each rat received a left sciatic nerve block (described below) under isoflurane anesthesia (nose-cone mask) using standard aseptic technique, without manipulation of the right sciatic nerve or other surgical insults. All investigators were blinded to treatment before data analysis, which occurred after the last experimental animal was euthanized.

\section{Sciatic nerve block treatment}

A standardized surgical technique, performed exclusively by investigator BAW for consistency, was used to expose the left sciatic nerve through a $1 \mathrm{~cm}$ skin incision in a manner similar to that reported by Brummett et al. (2008). ${ }^{7}$ One mg ropivacaine in $200 \mu \mathrm{L}$, or the same volume of preservative-free saline vehicle, was injected via 33 gauge needle (Vita-Needle, Waltham, MA) into the perineural subfascia of the left sciatic nerve under direct visualization with $6 \mathrm{x}$ loupe magnification. The surgical wounds were closed in a standard fashion with nylon suture. After emergence and recovery, animals were returned to their home cage. All surgeries were started after 5:00 am, completed by 8:00 am, and 2-3 surgical procedures were done per experimental day.

All rats received $200 \mu \mathrm{L}$ injections irrespective of preoperative weight. Ropivacaine injections were prepared from commercially available, preservative-free products used in patient care (diluted from 1\%, Naropin, Astra-Zeneca, Wilmington, DE). 


\section{Timeline of postoperative behavioral testing}

Rats were acclimated to the first postoperative behavioral test (Activity Monitor) for $15 \mathrm{~min}$ beginning at a mean of $34.0 \pm 0.1 \mathrm{~min}$ after injection. The purpose of activity testing was to assess sedative effects of residual volatile anesthetic and/or perineural ropivacaine.

After a 15 min activity assessment within the chamber, the rats underwent the RandallSelitto test exactly $50 \mathrm{~min}$ after injection (3 consecutive bilateral stimuli with an interstimulus interval of $1 \mathrm{~min}$ ). Rats where then acclimated for $5 \mathrm{~min}$ on the fenestrated stainless steel table prior to tactile analgesia-antinociception testing with monofilaments at $61.1( \pm$ 0.4 ) min after injection, using the up-down method. ${ }^{6}$ Tactile testing was performed as a frame of reference either for potential hypersensitivity at a later time point on the day of surgery, or to detect persistent sensory changes such as tactile hypersensitivity at the time of euthanasia. Monofilament testing was followed by heat nociception testing at $70.0 \pm 0.6 \mathrm{~min}$ with an inter-stimulus interval of $5 \mathrm{~min}$ ( 3 total radiant heat stimuli), after an initial $5 \mathrm{~min}$ acclimation on the heated table. Rats then underwent motor-proprioceptive testing on the rotarod at $89.0 \pm 0.1 \mathrm{~min}$ after injection. Rats were first acclimated to the rotarod device for 5 min prior to testing. A total of 3 test runs of no longer than 2 min duration (with 1 min of rest between tests) were conducted, and maximum speed achieved (rpm) and latency to falling (sec) were recorded. During preoperative training at a $20 \mathrm{rpm}$ limit, most rats were able to achieve maximum speed without falling from the rotating drum (ceiling effect). For day-of-surgery testing, rats were subjected to a rotating maximum speed of $40 \mathrm{rpm}$ (linear acceleration from the start point to $60 \mathrm{sec}$, then $40 \mathrm{rpm}$ plateau until $80 \mathrm{sec}$, then linear deceleration from 80 to $120 \mathrm{sec}$ ). Therefore, a speed achieved of $40 \mathrm{rpm}$ during testing indicated a minimum of $60 \mathrm{sec}$ of appropriate gait and proprioception.

Repeated Randall-Selitto testing on the day of surgery occurred exactly 10 min before repeat monofilament testing (i.e., at 170, 290, and $410 \mathrm{~min}$ ), and repeated monofilament testing occurred at exactly $3 \mathrm{hr}, 5 \mathrm{hr}$, and $7 \mathrm{hr}$ after injection. The first of three radiant heat stimuli for repeat thermal testing occurred exactly $8 \mathrm{~min}$ after the start of repeated monofilament testing (i.e., at 188,308 , and $428 \mathrm{~min}$ ), followed by similar $5 \mathrm{~min}$ inter-stimulus intervals. For convenience hereafter, and in figures $1-3$, the time points are simply represented as 1-3-5-7 hr after injection. Activity monitor and rotarod testing were repeated only once, at respectively 6.5 and $7.5 \mathrm{hr}$ after injection. After each of these 4 rounds of testing, rats were returned to their home cages and housing room with unrestricted access to food and water until the next round of testing.

\section{Behavioral training and testing before euthanasia}

Beginning on postoperative day one, rats were engaged in additional rotarod exercises to maintain their proprioceptive and ambulatory skills. The maximum rotating speed (40 rpm) and protocol (minimum three runs of no longer than 2 min duration, 1 min between runs) was the same as the day of surgery. This maintenance of rotarod skill exercise was performed on alternating days leading up to the day of euthanasia, with all rats exercised a total of 4 times. The goal of this "physical therapy" was to rule out deconditioning as a potential confounder in behavioral experiments conducted before euthanasia.

Rats were sacrificed on postoperative day 12-14. On the day before euthanasia, rats underwent a final round of tactile and thermal testing described above, as well as rotarod testing. The purpose of this additional testing was to determine if long-term effects of nerve block treatment (e.g.,hyperalgesia, persistent motor/proprioceptive dysfunction) were present. 


\section{Statistical analysis}

For exploratory data analysis, standard parametric (independent-samples t-test, one-way analysis of variance [ANOVA]) and non-parametric (Chi-square) statistics were used to assess differences between treatment groups. Two-way repeated-measures ANOVA was applied to assess the effects of treatment, time, and the interaction of treatment-time specifically for thermal and Randall-Selitto testing. All data were analyzed using SPSS v.17 Statistics software (SPSS, inc., Chicago, IL), and/or SigmaStat (Systat Software, Inc. Chicago, IL); $\mathrm{P}<0.05$ was considered to be statistically significant.

\section{Results}

A total of 28 rats were randomized; three rats were excluded from the study (failed block based on thermal testing at $1 \mathrm{hr}$, and not replaced). After exclusions, the final $\mathrm{n}=25$ : $\mathrm{VEH}$ $\mathrm{n}=11, \mathrm{RPV} \mathrm{n}=14$.

\section{Day of surgery/experimental sciatic nerve block behavioral testing}

\section{Activity monitor testing}

There were no significant differences between RPV versus VEH at either day-of-surgery time point measured (data not shown) with respect to level of activity after general anesthesia and sciatic nerve blockade.

\section{Mechanical anesthesia}

For Randall-Selitto testing at $1 \mathrm{hr}$ after injection, RPV was anesthetic $(249.5 \pm 0.5 \mathrm{~g})$, compared with VEH $(176.4 \pm 21.2 \mathrm{~g})$, as evidenced by a higher withdrawal threshold force at $1 \mathrm{hr}(\mathrm{P}<0.001)$ after injection. There were significant perineural anesthetic effects based on time and the interaction between treatment and time (each $\mathrm{P}<0.001)$. At baseline, treatments did not significantly differ between RPV $(131.9 \pm 11.0 \mathrm{~g})$ and VEH $(126.9 \pm 8.1$ g) with respect to withdrawal threshold. RPV withdrawal thresholds to Randall-Selitto testing returned to baseline and did not significantly differ from VEH by $3 \mathrm{hr}$ after block.

\section{Tactile anesthesia}

Upon testing with the monofilaments, there were no baseline differences between treatment groups related to the force required to elicit withdrawal in 50\% of the animals (i.e., ED50), which was $151 \pm 15 \mathrm{mN}$. RPV-treated rat hindlimbs were anesthetic to tactile stimuli up to the cut-off of $588 \mathrm{mN}$ at $1 \mathrm{hr}$ after nerve block injection, while for VEH-treated rats, the ED50 was $237 \pm 64 \mathrm{mN}(\mathrm{P}<0.001)$. RPV-treated rat hindlimbs did not differ from VEHtreated hindlimbs after the $1 \mathrm{hr}$ timepoint (e.g., RPV $170 \pm 46 \mathrm{mN}$ versus VEH $186 \pm 61 \mathrm{mN}$ at $3 \mathrm{hr}, \mathrm{P}=0.548$ ).

\section{Heat anesthesia and transient hyperalgesia}

Heat, tactile, and mechanical anesthesia were demonstrated $1 \mathrm{hr}$ after RPV treatment (i.e., failure to withdraw the hindpaw from the radiant heat stimulus by the $20 \mathrm{sec}$ cut-off). Heat hyperalgesia (shorter withdrawal latencies when compared with VEH) was seen at $3 \mathrm{hr}$ after RPV treatment (Figure 1); there was no coinciding tactile (Figure 2) or mechanical (Figure 3 ) hyperalgesia. A significant interaction between heat treatment and time was noted $(\mathrm{P}<0.001)$. Heat latency responses in both the RPV and VEH groups did not significantly differ at 5-7 hr or at sacrifice. Heat responses (RPV and VEH) significantly differed from baseline at each postoperative time point $(1,3,5$, and $7 \mathrm{hr}$ ). All latencies (RPV and VEH) returned to baseline by the time the rats were sacrificed at 2 weeks. The post hoc power 
values (alpha $=0.05$ ) computed for the significance of the time-treatment factor interaction, and for time only, were each 1.00 .

\section{Rotarod performance}

Eight of $11 \mathrm{VEH}$-treated rats achieved $40 \mathrm{rpm}$ at least once during the 3 separate rotarod tests at 90 min after injection (versus $1 / 14 \mathrm{RPV}$ treated animals, $\mathrm{P}<0.002$ ). At $7.5 \mathrm{hrs}$ post sciatic nerve block, there were no differences between RPV and VEH with respect to the achieved rotarod rpm or latency to fall time.

\section{Behavioral testing before euthanasia}

There were no differences between groups with respect to heat response latencies (Figure 1), monofilament testing (Figure 2), or rotarod performance immediately before euthanasia.

\section{Discussion}

\section{Overall summary of experimental findings}

This study verified preliminary findings of transient thermal hyperalgesia after rat sciatic nerve block with RPV versus VEH. Of particular interest, this transient thermal hyperalgesic response to heat was detectable in the setting of resolved mechanical sensory block (by the Randall-Selitto assay). The secondary finding of interest was that despite this transient thermal hyperalgesia, RPV sciatic blocks did not produce any detectable changes in sensory thresholds 12-14 days after nerve block injection; there was no evidence of nerve damage by nociceptive criteria, and all nociceptive responses and motor function returned to preoperative baseline.

\section{Modality specificity and nociceptive fiber specificity}

Different recovery responses with respect to mechanical (resolved block) versus thermal (transient heat hyperalgesia) stimuli in our study further illustrate the concept of modality specificity. Based on literature review of ropivacaine sciatic nerve blocks in rat ${ }^{8-15}$, we believe that this is the first report of transient thermal hypersensitivity to heat, irrespective of ongoing (or resolved) mechanical analgesia. To our knowledge, modality specificity has not been described in previous reports as heat hyperalgesia during recovery from perineural block from any local anesthetic.

\section{Clinical Application}

Rebound pain after a peripheral nerve block resolves is generally considered to be comprised of the mechanical-surgical pain that results from the resolution of a nerve block with unopposed nociceptive input. However, clinical experience ${ }^{4}$ has shown us that patients commonly describe an intense burning pain initially as the nerve block resolves. Whether these descriptions of burning pain correlate to our model's findings require further research, specifically to better determine differential time courses in vivo of heat hyperalgesia (or nociception) and mechanical nociception (or hyperalgesia). Such a model would likely include a panel of behavioral assessments as described herein or as previously described ${ }^{16,17}$, plus, the more detailed development of a model in which both nerve block resolution (such as in our model) and surgical pain ${ }^{18}$ can be separately evaluated.

\section{Study limitations}

A potential limitation in the present study is the influence of repeated heat stimulation of the hindpaw and sensitization of cutaneous nociceptors, thereby contributing to development of hyperalgesia. Rats treated with RPV were anesthetic $1 \mathrm{hr}$ after injection and received two thermal stimuli each of $20 \mathrm{sec}$ duration $5 \mathrm{~min}$ apart. The carefully calibrated mean maximum 
intensity $\left(49^{\circ} \mathrm{C}\right)$ may still have sensitized hindpaw nociceptors. However, gross inspection of the hindpaw during our experiments revealed no evidence of erythema or tissue damage after thermal testing. Additionally, thermal response latencies returned to baseline values at 3-5-7 hr, which argues against sensitization of nociceptors.

A second limitation would be the influence of repeated testing on the other behavioral tests (mechanosensory and motor-proprioception) used in this experiment. In order to prevent the influence of repeated testing on the same animal, a five minute interval between each of the three mechanosensory and three motor-proprioception tests was utilized. In order to better avoid potential confounding effects of repeated behavioral testing on the same animal, an additional control group of naïve animals (i.e., those that had no surgical manipulation) should be considered for future experiments.

A third limitation was that our study was specifically powered to detect heat hyperalgesia as a specific aim, verifying prior preliminary studies which demonstrated this unexpected finding. We employed the Randall-Selitto test to measure of mechanical anesthesiaanalgesia-antinociception; the study was not statistically powered to detect differences in these mechanical responses. Therefore, additional studies (with appropriate statistical power of both mechanical and thermal specific aims) may be necessary to confirm the finding of resolved (versus persistent) mechanical analgesia in the presence of thermal hyperalgesia.

\section{Conclusion}

During resolution of ropivacaine sciatic nerve blocks in rat, modality specificity was manifested as transient hypersensitivity to heat after mechanical block resolved. Further refinement of this rodent model is needed to show whether local anesthetics other than ropivacaine produce similar phenomena. Further pursuit of this model in the context of clinically used perineural analgesics (e.g., clonidine, buprenorphine, dexamethasone),) may also provide value in determining whether these adjuvants (in tandem or as multi-drug combinations with local anesthetics) either significantly prolong mechanical anesthesia, influence differential block specificity; and/or attenuate the unwanted transient hyperalgesia demonstrated after blocks with plain ropivacaine. However, it is important to recognize the limitations of such an animal model; one must use caution when extrapolating animal model behavorial findings to clinical experience.

\section{Acknowledgments}

Allison E. Garda, University of Pittsburgh (Pittsburgh, PA) for detailed laboratory scheduling, testing and data collection; Gerald F. Gebhart, PhD, Director, University of Pittsburgh Center for Pain Research (Pittsburgh, PA), for detailed guidance and scientific review throughout this project; Richard Day, PhD, University of Pittsburgh Graduate School of Public Health (Pittsburgh, Pennsylvania) for expertise in statistical consultation; Yan Xu, Ph.D., Vice Chair for Basic Research, University of Pittsburgh School of Medicine Department of Anesthesiology, for his review and critique of the manuscript; Pablo Brumovsky, MD, PhD, Michael Burcham, BS; Karen Hough, LVT, and Timothy McMurray (University of Pittsburgh Department of Anesthesiology, School of Medicine, Pittsburgh, Pennsylvania), for technical support.

\footnotetext{
This research is supported by the following: K01DA025146 (Dr. Williams), and 1 UL1 RR024153 (Dr. Williams), from the National Center for Research Resources (NCRR), a component of the National Institutes of Health (NIH), and NIH Roadmap for Medical Research. Regarding 1 UL1 RR024153, its contents are solely the responsibility of the authors and do not necessarily represent the official view of NCRR or NIH. Information on NCRR is available at http://www.ncrr.nih.gov/. Information on Re-engineering the Clinical Research Enterprise can be obtained from http://nihroadmap.nih.gov/clinicalresearch/overview-translational.asp. A special grant from the Office of the Senior Vice Chancellor for the Health Sciences, University of Pittsburgh is also acknowledged, as is additional material support from (i) University of Pittsburgh Department of Anesthesiology, and (ii) Pittsburgh Center for Pain Research. Dr. Williams received consulting fees from B.Braun USA (2010); B.Braun USA was not involved with the design or conduct of this study, and this manuscript or its contents were not made available to B.Braun USA prior to publication.
} 


\section{References}

1. Williams BA, Kentor ML, Vogt MT, et al. Femoral-sciatic nerve blocks for complex outpatient knee surgery are associated with less postoperative pain before same-day discharge: a review of 1200 consecutive cases from the period 1996 - 1999. Anesthesiology. 2003; 98:1206-1213. [PubMed: 12717143]

2. Zhang Y, Eger EI 2nd, Dutton RC, Sonner JM. Inhaled anesthetics have hyperalgesic effects at 0.1 minimum alveolar anesthetic concentration. Anesth Analg. 2000; 91:462-6. [PubMed: 10910869]

3. Tan T, Bhinder R, Carey M, Briggs L. Day-surgery patients anesthetized with propofol have less postoperative pain than those anesthetized with sevoflurane. Anesth Analg. 2010; 111:83-85. [PubMed: 19910624]

4. Williams BA, Bottegal MT, Kentor ML, Irrgang JJ, Williams JP. Rebound pain scores as a function of femoral nerve block duration after anterior cruciate ligament reconstruction: retrospective analysis of a prospective, randomized clinical trial. Reg Anesth Pain Med. 2007; 32:186-92. [PubMed: 17543812]

5. Anseloni VC, Ennis M, Lidow MS. Optimization of the mechanical nociceptive threshold testing with the Randall-Selitto assay. J Neurosci Methods. 2003; 131:93-7. [PubMed: 14659828]

6. Dixon WJ. Efficient analysis of experimental observations. Ann Rev Pharmacol Toxicol. 1980; 20:441-62. [PubMed: 7387124]

7. Brummett CM, Norat MA, Palmisano JM, Lydic R. Perineural administration of dexmedetomidine in combination with bupivacaine enhances sensory and motor blockade in sciatic nerve block without inducing neurotoxicity in rat. Anesthesiology. 2008; 109:502-511. [PubMed: 18719449]

8. Brummett CM, Padda AK, Amodeo FS, Welch KB, Lydic R. Perineural dexmedetomidine added to ropivacaine causes a dose-dependent increase in the duration of thermal antinociception in sciatic nerve block in rat. Anesthesiology. 2009; 111:1111-9. [PubMed: 19858875]

9. Hung Y-C, Chen C-Y, Lirk P, et al. Magnesium sulfate diminishes the effects of amide local anesthetics in rat sciatic-nerve block. Reg Anesth Pain Med. 2007; 32:288-95. [PubMed: 17720112]

10. Bouaziz H, Iohom G, Estebe JP, Campana WM, Myers RR. Effects of levobupivacaine and ropivacaine on rat sciatic nerve blood flow. Br J Anaesth. 2005; 95:696-700. [PubMed: 16183680]

11. Iohom G, Lan GB, Diarra DP, et al. Long-term evaluation of motor function following intraneural injection of ropivacaine using walking track analysis in rats. Br J Anaesth. 2005; 94:524-9. [PubMed: 15695548]

12. Kroin JS, Ling ZD, Buvanendran A, Tuman KJ. Upregulation of spinal cyclooxygenase-2 in rats after surgical incision. Anesthesiology. 2004; 100:364-9. [PubMed: 14739812]

13. Leduc C, Gentili ME, Estebe J-P, Le Corre P, Moulinoux J-P, Ecoffey C. The effect of local anesthetics and amitriptyline on peroxidation in vivo in an inflammatory rat model: preliminary reports. Anesth Analg. 2002; 95:992-6. [PubMed: 12351282]

14. Kohane DS, Sankar WN, Shubina M, Hu D, Rifai N, Berde CB. Sciatic nerve blockade in infant, adolescent, and adult rats: a comparison of ropivacaine with bupivacaine. Anesthesiology. 1998; 89:1199-208. [PubMed: 9822009]

15. Dyhre H, Lang M, Wallin R, Renck H. The duration of action of bupivacaine, levobupivacaine, ropivacaine and pethidine in peripheral nerve block in the rat. Acta Anaesthesiol Scand. 1997; 41:1346-52. [PubMed: 9422304]

16. Feldman HS, Covino BG. Comparative motor-blocking effects of bupivacaine and ropivacaine, a new amino amide local anesthetic, in the rat and dog. Anesth Analg. 1988; 67:1047-52. [PubMed: 3189893]

17. Thalhammer JG, Vladimirova M, Bershadsky B, Strichartz GR. Neurologic evaluation of the rat during sciatic nerve block with lidocaine. Anesthesiology. 1995; 82:1013-25. [PubMed: 7717536]

18. Hamalainen MM, Subieta A, Arpey C, Brennan TJ. Differential effect of capsaicin treatment on pain-related behaviors after plantar incision. J Pain. 2009; 10:637-45. [PubMed: 19386553] 


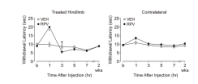

Figure 1. Heat anesthesia-analgesia followed by shorter latencies to heat after sciatic nerve block with ropivacaine

This is an illustration of nociceptive responses of the treated (left) hindlimb to heat stimuli as a function of treatment and time $(\mathrm{P}<0.001)$. The last time point on the $\mathrm{X}$-axis represents behavioral testing before rats were euthanized (postoperative day 12-14). The ropivacaine (RPV) group showed anesthesia at $1 \mathrm{hr}$ after injection $(\mathrm{P}<0.001)$, compared to the vehicle group (VEH), followed by a shorter response latency to heat stimuli $(\dagger \mathrm{P}=0.001)$ at $3 \mathrm{hr}$ after injection. There was no evidence of transient heat hyperalgesia at 5-7 hr, or long-term heat hyperalgesia at 2 weeks. 


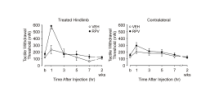

Figure 2. Tactile withdrawal threshold $(\mathrm{mN})$ to von Frey monofilament testing after sciatic nerve block with ropivacaine

This is an illustration of nociceptive responses of the treated (left) hindlimb to tactile stimuli as a function of treatment and time. The last time point (lon the $\mathrm{X}$-axis represents behavioral testing before rats were euthanized (postoperative day 12-14). The ropivacaine (RPV) group showed anesthesia at $1 \mathrm{hr}$ after injection $(\mathrm{P}<0.001)$, compared to the vehicle group (VEH). There were no statistically significant differences in tactile withdrawal threshold $(\mathrm{mN})$ to von Frey monofilament testing between the RPV and VEH groups at any other time point. 


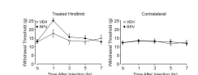

Figure 3. Mechanical anesthesia after sciatic nerve block with ropivacaine as demonstrated with Randall-Selitto testing

This is an illustration of mechanical anesthesia demonstrated with Randall-Selitto testing as a function of treatment and time. The last time point (on the X-axis) represents behavioral testing before rats were euthanized (post operative day 12-14). At baseline, treatments did not significantly differ between RPV $(131.9 \pm 11.0 \mathrm{~g})$ and VEH $(126.9 \pm 8.1 \mathrm{~g})$ with respect to withdrawal threshold. At $1 \mathrm{hr}$ after injection, the ropivacaine (RPV) group was anesthetic $(249.5 \pm 0.5 \mathrm{~g})$ compared with VEH $(176.4 \pm 21.2 \mathrm{~g})$ as evidenced by a higher withdrawal threshold force $(\mathrm{P}<0.001)$. RPV withdrawal thresholds to Randall-Selitto testing returned to baseline and did not significantly differ from VEH by $3 \mathrm{hr}$ after block. 